\title{
Disfunção Diastólica em Pacientes Diabéticos Normotensos, Independente da Presença de Microangiopatia
}

\author{
Diastolic Dysfunction in Diabetic Normotensive Patients, Regardless of the Presence \\ of Microangiopathy
}

\author{
João Carlos Ferreira Braga, Fábio Villaça Guimarães Filho, Carlos Roberto Padovani, \\ Beatriz B. Matsubara \\ Faculdade Estadual de Medicina de Marília e Faculdade de Medicina de Botucatu \\ Marília, SP - Botucatu, SP
}

\section{Objetivo}

Avaliar as alterações Doppler-ecocardiográficas de pacientes normotensos, com diabetes mellitus tipo II, na presença ou ausência de sinais de microangiopatia.

\section{Métodos}

Pacientes com diabetes mellitus tipo II foram submetidos a exames de fundo de olho contrastado com fluoresceína e dosagem de microalbuminúria para diagnóstico de microangiopatia, e divididos em dois grupos: DMII (pacientes sem microangiopatia, $n=19$ ) e $D M+A$ (pacientes com microangiopatia, $n=13$ ). Todos foram submetidos a Doppler-ecocardiografia e os resultados comparados com normotensos de mesmo sexo e idade (grupo $C, n=20$ ), aplicando-se a ANOVA, seguida de teste de Tukey. Em todas as comparações, adotou-se como nível de significância $p<0,05$.

\section{Resultados}

Não houve diferenças entre os grupos quanto aos indicadores de função sistólica ou massa do ventrículo esquerdo. Foram observadas diferenças compatíveis com disfunção diastólica nos dois grupos de diabéticos, independente da presença de microangiopatia, que exibiram valores significativamente maiores dos tempos de relaxamento isovolumétrico do ventrículo esquerdo (TRIVE, $\mathrm{ms}$ ): (DMII $=97 \pm 22,2 ; D M+A=107 \pm 28,2$ e $C=80 \pm 10,7 ; p<0,05$ ), e menores das velocidades máximas da onda de enchimento ventricular rápido $(E, c m / s):(D M I I=69 \pm 17,5 ; D M+A=75 \pm 19,7$ e $C=84 \pm 14,5, p<0,05$ entre DMII e C). Sem diferença entre os grupos quanto à razão $\mathrm{E} / \mathrm{A}$.

\section{Conclusão}

Pacientes normotensos com diabetes mellitus tipo II e sem sinais clínicos de comprometimento cardiovascular, apresentaram sinais de disfunção diastólica não associados à presença de microangiopatia.

\section{Palavras-chave}

ecocardiograma, ventrículo esquerdo, cardiomiopatia, diabetes mellitus

\section{Objective}

To assess the Doppler-echocardiographic changes in normotensive patients with type II diabetes mellitus, in the presence or absence of signs of microangiopathy.

\section{Methods}

Patients with type II diabetes mellitus were submitted to funduscopy contrasted with fluorescein and dosage of microalbuminuria for diagnose of microangiopathy and divided into two groups: DMII (patients without microangiopathy, $\mathrm{n}=19$ ) and $\mathrm{DM}+\mathrm{A}$ (patients with microangiopathy, $n=13$ ). All of them were submitted to a Doppler-echocardiography and the results were compared with normotensive patients of same sex and age (group C, $n=20$ ), by using the ANOVA, followed by the test of Tukey. In all comparisons the significance level $p<0.05$ was adopted.

\section{Results}

There were no differences among the groups regarding the systolic function indicators or left ventricular mass. Differences compatible with diastolic dysfunction in the two groups of diabetic were observed, regardless of the presence of microangiopathy, which showed significantly higher values of the times of isovolumetric relaxation of the left ventricle (TIRLV, ms): (DMII= $97 \pm 22.2 ; \mathrm{DM}+\mathrm{A}=107 \pm 28.2$ and $\mathrm{C}=80 \pm 0.7 ; p<0.05)$, and lower values of the maximum speeds of the wave of fast ventricular filling $(E, c m / s)$ : $(D M I I=69 \pm 17.5 ; D M+A=75 \nexists 19.7$ and $C=84 \nexists 14.5, p<0.05$ between DMII and $C$ ). There was no difference among the groups concerning the E/A rate.

\section{Conclusion}

Normotensive patients with type II diabetes mellitus and without clinical signs of cardiovascular compromising showed signs of diastolic dysfunction, non-associated to the presence of microangiopathy.

\section{Key words}

echocardiogram, left ventricle, cardiomiopathy, diabetes mellitus 
Evidências clínicas, epidemiológicas e histopatológicas indicam a existência de uma cardiopatia específica relacionada ao diabetes mellitus $^{1}$. De fato, avaliações não invasivas da função cardíaca em indivíduos diabéticos, freqüentemente, mostram a existência de anormalidades de ambas as funções sistólica e diastólica do ventrículo esquerdo, as quais podem ser evidenciadas por uma variedade de métodos diagnósticos. No entanto, a despeito do uso comum do termo cardiomiopatia diabética, ainda existe considerável debate a respeito da exata natureza e causa da disfunção cardíaca encontrada em diabéticos não portadores de aterosclerose coronariana e hipertensão arterial, que são condições patológicas freqüentemente associadas ao diabete millitus e que podem levar à disfunção cardíaca, segundo a literatura.

A existência da cardiomiopatia diabética foi primeiramente sugerida por Rubler e cols. ${ }^{1}$, que descreveram hipertrofia e fibrose miocárdica, além de proliferação endotelial e subendotelial proeminentes, indicando que as alterações nos pequenos vasos poderiam estar envolvidas na patogênese da disfunção miocárdica.

Desde essa descrição inicial, e até o momento, ainda persistem as controvérsias quanto à relativa importância da doença nos pequenos vasos, da fibrose intersticial e dos distúrbios metabólicos na patogênese da cardiomiopatia diabética ${ }^{2-15}$.

Rossen ${ }^{15}$ argumentou que, embora a correlação da cardiomiopatia com microangiopatia seja incerta, ela pode existir, tendo em vista as semelhanças entre as anormalidades na função microvascular coronariana observadas no diabete millitus e na cardiomiopatia dilatada idiopática.

Gutierrez e Higuchi ${ }^{14}$, em nosso meio, aceitam três hipóteses para explicar o que chamam de eventual efeito causal do diabetes quanto à doença cardíaca: 1) microangiopatia; 2) ação direta sobre a fibra cardíaca, dos distúrbios metabólicos provocados pela hiperglicemia, entre outros fatores; 3 ) alterações da matriz extracelular, provocadas pelo maior grau de glicosilação de seus componentes, com conseqüente mudanças da estrutura muscular.

Até o presente, os estudos que compararam a função ventricular de pacientes diabéticos com ou sem microangiopatia ${ }^{10,15-20}$ apresentaram resultados conflitantes. Recentemente Liu e cols. ${ }^{21}$ encontraram relação entre a presença de perda urinária de albumina e disfunção diastólica, em três grupos analisados: ausência de albuminúria, microalbuminúria e macroalbuminúria. Apesar das diferenças entre os grupos, a análise multivariada mostrou albuminúria como variável independentemente associada com disfunção sistólica e diastólica, após ajuste para idade, sexo, índice de massa corpórea, pressão arterial sistólica, duração do diabete millitus, doença arterial coronariana, e índice de massa do ventrículo esquerdo. Uma vez que a microalbuminúria é um marcador de disfunção endotelial na arteríola glomerular, Bell122 achou válido postular que a disfunção endotelial no miocárdio causa aumento de cicatrização e rigidez, considerando, ainda, que este teste urinário de baixo custo poderia antecipar um exame de ecodopplercardiografia, que, embora mais sensível para detecção de disfunção diastólica, tem maior custo. Documentada a disfunção diastólica, deveria ter inicio a terapia visando prevenir a progressão da doença para insuficiência cardíaca.

Tendo em vista que o exame ecocardiográfico é descrito como método sensível para detecção precoce da cardiomiopatia diabéti$\mathrm{Ca}^{8}$, o presente estudo tem por objetivo identificar se a presença de microangiopatia está associada a alterações estruturais e funcionais cardíacas em diabéticos não hipertensos, e assintomáticos

\section{Métodos}

Todos os procedimentos foram submetidos e aprovados por Comitê de Ética em Pesquisa Envolvendo Seres Humanos.

Foi realizado um estudo transversal de caso-controle envolvendo 32 pacientes com diabetes mellitus, com idades entre 40 e 65 anos e 20 voluntários normais, com idade e sexo comparáveis. Todos os participantes do estudo passaram por avaliação clínica completa com o cardiologista.

Foram critérios de exclusão: pressão arterial sistólica $\geq 140 \mathrm{mmHg}$ ou pressão arterial diastólica $\geq 90 \mathrm{mmHg}$; sinais ou sintomas de doença cardiorespiratória; uso de drogas de ação cardiovascular; janela acústica imprópria para a análise do ecocardiograma.

Os pacientes diabéticos foram submetidos a exames laboratoriais para avaliação de função renal e de perfil lipídico e, também, a exame de fundo de olho contrastado com fluoresceína, e de urina coletada em $12 \mathrm{~h}$ para detecção de microalbuminúria. 0 diagnóstico de retinopatia e/ou de microalbuminúria foi o critério para a definição da presença de microangiopatia.

0 grupo DM +A foi composto de pacientes com sinais de microangiopatia $(n=13)$; o grupo DM por pacientes sem microangiopatia ( $n=19)$; e o grupo controle de voluntários clinicamente normais $(n=20)$.

0 exame de fundo de olho constou de oftalmoscopia direta e indireta sob midríase; retinografia com luz anéritra, angiografia fluoresceínica, por meio da injeção de $2,5 \mathrm{ml}$ de fluoresceína sódica a $10 \%$, via endovenosa. Para a documentação da angiografia fluoresceínica e da retinografia foi utilizado o equipamento marca Topcon, modelo TRC-FE e filme Kodak Tri-X Pam de 400 ASA. 0 diagnóstico de retinopatia foi baseado na presença de pelo menos um microaneurisma em um dos olhos ou na de outra alterações, como hemorragias, exsudatos duros, exsudatos algodonosos e proliferação fibrosvascular ${ }^{23}$.

A excreção urinária de albumina foi determinada por meio de turbidimetria, utilizando-se 0 equipamento Turbidimeter $^{\circledR}$ da Behring e os reativos Turbiquant ${ }^{\circledR}$, de sensibilidade de 0,3 micrograma/ml. 0 exame foi realizado em urina colhida por $12 \mathrm{~h} \mathrm{em}$ frasco estéril e sem preservativos. As amostras contendo bacteriúria $>10^{5} / \mathrm{mm}^{3}$ eram desprezadas. 0 resultado foi considerado positivo para os valores maiores que 15 microgramas/minuto.

Todos os procedimentos foram realizados por um único ecocardiografista experiente que não tinha conhecimento do grupo a que pertenciam os indivíduos. Os exames foram efetuados por meio equipamento ATL Apogee CX 200, dotado de transdutor ultra-sônico multifrequêncial de 2,0-3,5 MHz e sistema de registro de imagens. Durante o procedimento, o paciente permanecia em decúbito lateral esquerdo, com o membro superior esquerdo ligeiramente fletido sob a cabeça. Uma derivação eletrocardiográfica foi continuamente monitorada.

As imagens foram obtidas seguindo-se as recomendações da American Society of Echocardiography ${ }^{24}$, a partir de cortes ecocardiográficos convencionais. Foram medidas em seqüência: dimensão sistólica final da via de saída do VE (VSVE), diâmetro diastólico ântero-posterior do átrio esquerdo (AE), diâmetro diastólico final (VEd), diâmetro sistólico final (VEs), espessuras diastólicas do SIV e da parede posterior do VE (PP). Essas dimensões foram utilizadas para o cálculo de: 
Índice de massa VE (IMVE, gr/m²) = $\frac{\left\{\left[(\mathrm{SIVd}+\text { PPVEd }+ \text { DdVE })^{3}-\text { DDVE }^{3}\right] \times 1.04\right\}-13.6}{\mathrm{SC}}$

Fração de ejeção (FE) =

$[7 /(2.4+\mathrm{DdVE})] \times \mathrm{DdVE}^{3}-[7 /(2.4+\mathrm{DsVE})] \times \mathrm{DsVE}^{3}$

$[7 /(2.4+\mathrm{DdVE})] \times \mathrm{DdVE}^{3}$

Porcentagem de variação do diâmetro ventricular $(\% \Delta \mathrm{D})=$ [(VEd - VEs) / Ved] x 100.

As avaliações dos fluxos seguiram as orientações do Consenso Canadense ${ }^{25}$ para as medidas Doppler-ecocardiográficas, obtendo-se as seguintes variáveis: $\mathrm{c} 1$ ) velocidade máxima de enchimento ventricular rápido ( $p i c o$ da onda $E, \mathrm{~cm} / \mathrm{s}$ ); $\mathrm{c} 2$ ) velocidade máxima de enchimento tardio, após a contração atrial (pico da onda $A$, $\mathrm{cm} / \mathrm{s}$ ); c3) tempo de desaceleração da onda $E$ (TDE, ms), correspondente ao intervalo de tempo entre o pico da onda $E$ e a sua extrapolação para a linha de base. Ainda com o Doppler pulsátil, obteve-se as curvas simultâneas de fluxo nas vias de saída e de entrada do VE. O intervalo de tempo entre o final do fluxo sistólico e o início do fluxo transmitral correspondeu ao tempo de relaxamento isovolumétrico do VE (TRIV). A análise do fluxo sistólico na VSVE permitiu o cálculo da seguintes variáveis: índice sistólico $(\mathrm{IS}, \mathrm{mL} / \mathrm{SC})=\left(\mathrm{VSVE}^{2} \times 0,785 \times\right.$ IVSVE$) / S C$, onde VSVE é a medida da via de saída do VE, IVSVE é a integral do fluxo sistólico na VSVE e SC é a superfície corpórea; débito cardíaco (DC, L/ $\min )=V S \times F C$.

As comparações entre os grupos foram efetuadas por meio da ANOVA, seguida do teste de Tukey. Em todos os casos, considerou-se o nível de significância $p<0,05$.

\section{Resultados}

Diabéticos com microangiopatia apresentaram valores médios de microalbuminúria de $36 \mathrm{mcg} / \mathrm{min}$. Do mesmo modo as altera- ções mais freqüentes observadas no exame contrastado de fundo de olho foram a presença de microaneurismas, quadro classificado como retinopatia diabética não proliferativa ${ }^{23}$. Dos 13 pacientes do grupo $\mathrm{DM}+\mathrm{A}, 9$ apresentaram alteração isolada de fundo de olho, 2 somente microalbuminúria e em 2 ambas as alterações.

$\mathrm{Na}$ tabela I são apresentadas a idade, o peso corporal, a superfície corpórea e as variáveis hemodinâmicas dos grupos diabetes mellitus com microangiopatia $(D M+A)$, diabetes mellitus sem microangiopatia (DM) e controle (C). São apresentados os valores das médias e seus respectivos desvios-padrão e os resultados do teste estatístico. Quanto às variáveis idade, peso e superfície corpórea, os 3 grupos foram estatisticamente semelhantes entre si. A comparação dos dados hemodinâmicos evidenciou que os grupos não são diferentes quanto à pressão arterial sistólica e pressão arterial diastólica. Em relação à freqüência cardíaca, o grupo DM apresentou valor, estatisticamente significante, maior que 0 grupo C (74 $\pm 10,30$ bpm vs $65 \pm 9,40 \mathrm{bpm}, \mathrm{p}<0,05)$.

Na tabela II, são observadas as variáveis morfométricas cardíacas, normalizadas para a superfície corporal, obtidas por meio do ecocardiograma modo $\mathrm{M}$, dos grupos diabetes mellitus com microangiopatia $(D M+A)$, diabetes mellitus sem microangiopatia (DM) e controle (C). Não foram observadas diferenças significantes entre esses 3 grupos.

Na tabela III encontram-se as variáveis de função sistólica, obtidas pela Doppler-ecocardiografia, dos três grupos estudados, não havendo diferenças estatísticas entre eles e na tabela IV as variáveis da função diastólica. A velocidade máxima do enchimento ventricular rápido $(E)$ foi semelhante nos grupos $D M+A(75 \pm$ $19,70 \mathrm{~cm} / \mathrm{s})$ e DM $(69 \pm 17,50 \mathrm{~cm} / \mathrm{s})$. O valor obtido nesse último grupo foi significantemente menor do que o observado no grupo controle $(84 \pm 14,50 \mathrm{~cm} / \mathrm{s})$. 0 tempo de relaxamento isovolumétrico do ventrículo esquerdo (TRIVE) apresentou valores estatisticamente

\begin{tabular}{|c|c|c|c|c|}
\hline \multirow[t]{2}{*}{ Variável } & \multicolumn{3}{|c|}{ Grupos } & \multirow{2}{*}{$\begin{array}{l}\text { Resultado do } \\
\text { teste estatístico }\end{array}$} \\
\hline & $\mathrm{DM}+\mathrm{A}$ & DM & $\mathrm{C}$ & \\
\hline Id(anos) & $55,00 \pm 6,90$ & $50,90 \pm 8,47$ & $51,20 \pm 9,41$ & $1,06(p>0,05)$ \\
\hline$P C(K g)$ & $71,60 \pm 11,24$ & $72,30 \pm 12,54$ & $68,50 \pm 14,97$ & $0,45(p>0,05)$ \\
\hline $\mathrm{SC}\left(\mathrm{Kg} / \mathrm{m}^{2}\right)$ & $1,81 \pm 0,18$ & $1,79 \pm 0,18$ & $1,76 \pm 0,24$ & $0,27(p>0,05)$ \\
\hline PAS (mmHg) & $120 \pm 13,60$ & $\pm 11,50$ & $112,00 \pm 10,57$ & $2,11(p>0,05)$ \\
\hline PAD (mmHg) & $79,00 \pm 8,20$ & $\pm 7,90$ & $76,00 \pm 5,90$ & $1,24(p>0,05)$ \\
\hline $\mathrm{FC}(\mathrm{bpm})$ & $71,00 \pm 8,40^{(a b)}$ & $74,00 \pm 10,30^{(b)}$ & $65,00 \pm 9,40^{(a)}$ & $4,82(p<0,05)$ \\
\hline
\end{tabular}

\begin{tabular}{|c|c|c|c|c|}
\hline \multirow[t]{2}{*}{ Variável } & \multicolumn{3}{|c|}{ Grupos } & \multirow{2}{*}{$\begin{array}{l}\text { Resultado do } \\
\text { teste estatístico }\end{array}$} \\
\hline & $\mathrm{DM}+\mathrm{A}$ & DM & $C$ & \\
\hline $\mathrm{AE} / \mathrm{SC}\left(\mathrm{cm} / \mathrm{m}^{2}\right)$ & $1,81 \pm 0,21$ & $1,90 \pm 0,25$ & $1,89 \pm 0,27$ & $0,56(p>0,05)$ \\
\hline $\mathrm{VEd} / \mathrm{SC}\left(\mathrm{cm} / \mathrm{m}^{2}\right)$ & $2,52 \pm 0,20$ & $2,56 \pm 0,45$ & $2,67 \pm 0,23$ & $0,95(p>0,05)$ \\
\hline $\mathrm{VEs} / \mathrm{SC}\left(\mathrm{cm} / \mathrm{m}^{2}\right)$ & $1,53 \pm 0,17$ & $1,50 \pm 0,30$ & $1,51 \pm 0,22$ & $0,06(p>0,05)$ \\
\hline IMVE $\left(\mathrm{g} / \mathrm{m}^{2}\right)$ & $90,60 \pm 26,80$ & $90,70 \pm 36,00$ & $80,10 \pm 22,00$ & $0,83(p>0,05)$ \\
\hline
\end{tabular}




\begin{tabular}{|c|c|c|c|c|}
\hline \multirow[t]{2}{*}{ Variável } & \multicolumn{3}{|c|}{ Grupos } & \multirow{2}{*}{$\begin{array}{l}\text { Resultado do } \\
\text { teste estatísticc }\end{array}$} \\
\hline & $\mathrm{DM}+\mathrm{A}$ & DM & $C$ & \\
\hline Delta d & $0,39 \pm 0,06$ & $0,41 \pm 0,05$ & $0,43 \pm 0,07$ & $1,91(p>0,05)$ \\
\hline $\mathrm{FE}$ & $0,69 \pm 0,07$ & $0,72 \pm 0,05$ & $0,74 \pm 0,07$ & $2,23(p>0,05)$ \\
\hline $\mathrm{DC}(\mathrm{L} / \mathrm{min})$ & $4,25 \pm 0,98$ & $4,20 \pm 1,26$ & $3,94 \pm 1,29$ & $0,33(p>0,05)$ \\
\hline $\mathrm{IS}\left(\mathrm{mL} / \mathrm{m}^{2}\right)$ & $32,80 \pm 6,00$ & $32,30 \pm 10,80$ & $34,00 \pm 6,90$ & $0,23(p>0,05)$ \\
\hline $\operatorname{SSF}\left(\mathrm{g} / \mathrm{cm}^{2}\right)$ & $51,60 \pm 12,20$ & $49,40 \pm 12,90$ & $47,00 \pm 14,10$ & $0,49(p>0,05)$ \\
\hline
\end{tabular}

\begin{tabular}{|c|c|c|c|c|}
\hline \multirow[t]{2}{*}{ Variável } & \multicolumn{3}{|c|}{ Grupos } & \multirow{2}{*}{$\begin{array}{l}\text { Resultado do } \\
\text { teste estatístico }\end{array}$} \\
\hline & DM + Angio & $\overline{D M}$ & $\bar{C}$ & \\
\hline$E(\mathrm{~cm} / \mathrm{seg})$ & $75,00 \pm 19,70^{\text {(ab) }}$ & $69,00 \pm 17,50^{\text {(a) }}$ & $84,00 \pm 14,50^{\text {(b) }}$ & $4,15(p<0,05)$ \\
\hline $\mathrm{A}(\mathrm{cm} / \mathrm{seg})$ & $80,00 \pm 16,20$ & $71,00 \pm 16,70$ & $75,00 \pm 14,90$ & $1,52(p>0,05)$ \\
\hline$E / A$ & $0,94 \pm 0,28$ & $0,99 \pm 0,26$ & $1,16 \pm 0,30$ & $2,69(p>0,05)$ \\
\hline TDE (mseg) & $181,00 \pm 51,00$ & $192,00 \pm 36,40$ & $188,00 \pm 42,10$ & $0,31(p>0,05)$ \\
\hline TRIVE (mseg) & $107,00 \pm 28,20^{(b)}$ & $97,00 \pm 22,20^{(b)}$ & $80,00 \pm 10,70^{\text {(a) }}$ & $7,61(p<0,05)$ \\
\hline
\end{tabular}

semelhantes nos pacientes DM + A (107 $\pm 28,20 \mathrm{~ms})$ e DM $(97 \pm$ $22,20 \mathrm{~ms}$ ). Ambos os grupos foram diferentes do grupo controle, que apresentou valor significantemente menor ( $80 \pm 10,70 \mathrm{~ms})$.

\section{Discussão}

A ausência de diferenças entre os grupos de pacientes, com relação às variáveis morfométricas e de função sistólica, não foi surpresa. Os critérios de inclusão e de exclusão foram bastante específicos e restringiram a amostra de pacientes. Esse fato, embora tenha contribuído para diminuir o poder de evidenciar diferenças potenciais entre os grupos, foi concordante com a proposta de avaliar a associação entre presença de sinais de microangiopatia diabética e alterações morfofuncionais cardíacas em grupos semelhantes em todos os demais aspectos, exceto a lesão de pequenos vasos. Também foi necessário restringir as análises aos pacientes diabéticos não portadores de outras doenças cardiovasculares. Aliás, esse tem sido o problema enfrentado por pesquisadores interessados no assunto. Por exemplo, vários estudos com casuística maior concluíram sobre disfunção ventricular esquerda associada ao diabetes, incluindo no mesmo grupo pacientes normotensos e hipertensos ${ }^{16,26,27}$. Cosson e cols ${ }^{8}$ atribuíram os resultados contraditórios publicados à falta de homogeneidade das populações estudadas e de uniformidade dos índices ecocardiográficos utilizados. Assim, nossas conclusões são aplicáveis apenas a grupos de indivíduos com características semelhantes aos avaliados no presente estudo.

0 estudo de Framingham ${ }^{22}$ mostrou que mulheres diabéticas apresentavam massa de ventrículo esquerdo $10 \%$ maior que as não diabéticas. 0 estudo Tayside ${ }^{28}$ mostrou que a hipertrofia ventricular esteve presente em $32 \%$ dos diabéticos normotensos, os angiotensina e não tinham doença coronariana. Além disso, mulheres hipertensas e diabéticas apresentaram maior grau de hipertrofia ventricular esquerda e aumento do átrio esquerdo, quando comparadas a hipertensas não diabéticas ${ }^{29}$. Enquanto a fibrose miocárdica parece estar associada à hiperglicemia, a hipertrofia ventricular esquerda tem sido mais relacionada à síndrome de resistência à insulina ${ }^{30,31}$. Embora a hipertrofia ventricular esquerda seja mais prevalente em diabéticos e, tal fato seja relacionado com disfunção ventricular ${ }^{22,32}$, em nossa casuística, não houve variação da massa ventricular esquerda.

A despeito da função sistólica preservada em repouso, considerável proporção desses pacientes apresentaram alterações diastólicas ao exame Doppler-ecocardiográfico. Esses achados estão em acordo com as observações clínicas de outros autores que mostraram disfunção diastólica precedendo a disfunção sistólica, na evolução das alterações cardíacas de pacientes com diabetes mellitus ${ }^{8,32,33}$.

0 estudo de Framingham convincentemente mostrou que pacientes diabéticos apresentam risco adicional para o desenvolvimento de cardiomiopatia e insuficiência cardíaca ${ }^{34}$. No entanto, não ficou completamente esclarecida a natureza dessa associação relatada naquele clássico estudo epidemiológico. A identificação de que muitos dos pacientes com sinais clínicos de falência cardíaca apresentavam, objetivamente, função sistólica preservada, provocou enorme interesse dos pesquisadores e clínicos sobre o estado

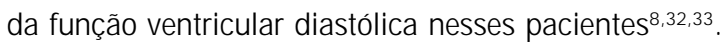

Esse comprometimento diastólico que precede as alterações sistólicas na evolução das alterações funcionais do coração diabético tem sido observado mesmo na ausência de doença arterial coronariana e hipertrofia ventricular esquerda. Diversos relatos têm mostrado prevalências de 30 a $60 \%$ de disfunção diastólica em indivíduos diabéticos bem controlados e normotensos $22,32,35-37$. 
Um achado interessante no presente estudo foi o aumento do tempo de relaxamento isovolumétrico nos pacientes diabéticos, quando comparado aos controles. Apesar desse aumento, em média, não ser de magnitude suficiente para extrapolar os valores considerados normais, pode-se assumir que algum grau de comprometimento do processo de relaxamento miocárdico deve estar em curso nesse pacientes.

O relaxamento miocárdico ocorre em conseqüência à retirada do íon $\mathrm{Ca}^{2+}$ do citoplasma, para dentro do retículo sarcoplasmático, após a contração miocárdica, num processo complexo, ativo, com grande consumo de ATP e envolvendo inúmeras proteínas. Portanto, o prolongamento do tempo de relaxamento miocárdico pode ser 0 resultado de uma grande quantidade de potenciais alterações subcelulares que poderiam prejudicar o relaxamento.

O prolongamento do tempo de relaxamento miocárdico, influência o enchimento ventricular rápido e poderia ser o mecanismo subjacente à observação de valores diminuídos no pico da onda $E$. 0 enchimento ventricular depende fundamentalmente do gradiente de pressão atrioventricular, durante essa fase do ciclo cardíaco. Esse gradiente de pressão é diretamente influenciado pela pressão intra-atrial e, portanto, pelo seu volume, e inversamente influenciado pela pressão intraventricular. Assim, o relaxamento miocárdico retardado ou incompleto, promove aumento na pressão diastólica ventricular e redução no gradiente transmitral e diminuição da velocidade de fluxo diastólico inicial. Existe, ainda, a possibilidade de que alterações intersticiais miocárdicas, com aumento da concentração de colágeno, tenham participado do processo de indução da disfunção diastólica ${ }^{38}$.

Contrariamente ao esperado, não observamos diferenças significantes entre os dois grupos de pacientes diabéticos. Embora na literatura a presença de albuminúria em indivíduos diabéticos identifica alto risco de presença de doença cardiovascular, os nossos dados sugerem, que sua presença não está relacionada com disfunção diastólica do ventrículo esquerdo, alteração essa considerada manifestação inicial da cardiomiopatia diabética21,33. Nesse aspecto, é relevante considerar o tamanho da amostra. Isto é, diferenças entre os grupos poderiam ser demonstradas, estatisticamente, se 0 número de pacientes fosse maior. No entanto, dada a semelhança entre os valores médios dos índices de função diastólica nos grupos $\mathrm{DM}$ e $\mathrm{DM}+\mathrm{A}$, somente a inclusão de centenas de pacientes no estudo permitiria a demonstração. Apesar dessas limitações, consideramos válida a divulgação dos resultados do presente estudo, tendo em vista que estudos semelhantes podem ser realizados e, em conjunto, permitam no futuro resolver esta questão relevante a respeito da fisiopatologia da cardiomiopatia do diabético.

Finalmente, consideramos de grande relevância a observação de que pacientes diabéticos normotensos e sem sinais clínicos de doença cardíaca apresentam alterações sugestivas de disfunção diastólica, quando comparados com seus controles não diabéticos. Estes resultados reforçam a necessidade de estudos adicionais semelhantes, buscando esclarecer a fisiopatologia, as formas de prevenção e o tratamento desta disfunção.

\section{Referências}

1. Rubler S, Dlugash J, Yuceoglu YZ, Kumral T, Branwood AW, Grishman A. New type of cardiomyopathy associated with diabetic glomerulosclerosis. Am J Cardiol 1972;30:595-602.

2. Uusitupa MI, Mustonen J N, Airaksinen KE. Diabetic heart muscle disease. Ann Med 1990;22:377-86.

3. AronsonD, Rayfield E. Diabetes. In: Topol E, editor.Textbook of Cardiovascular Medicine. 2nd edition. Philadelphia, PA; Lippincott Williams \& Wilkins, 2002:171-94.

4. Nesto RW, Libby P. Diabetes mellitus and the cardiovascular system. In: Braunwald E, Zipes DP, Libby P, editors. Heart Disease. A Textbook of Cardiovascular Medicine. $6^{\text {th }}$ edition. Philadelphia, PA; WB Saunders, 2001:2133-50.

5. Uusitupa MI, Mustonen J N, Laakso M et al. Impairment of diastolic function in middle-aged type 1 (insulin-dependent) and type 2 (non-insulin-dependent) diabetic patients free of cardiovascular disease. Diabetologia 1988;31:783-91.

6. Uusitupa MI, Siitonen O, Pyorala K, Lansimies E. Left ventricular function in newly diagnosed non-insulin-dependent (type 2 ) diabetics evaluated by systolic time intervals and echocardiography. Acta Med Scand 1985;217:379-88.

7. Vered A, Battler A, Segal $P$ et al. Exercise-induced left ventricular dysfunction in young men with asymptomatic diabetes mellitus (diabetic cardiomyopathy). Am J Cardiol 1984; 54:633-7.

8. Cosson S, Kevorkian J. Left ventricular diastolic dysfunction: an early sign of diabetic cardiomypathy ? Diabetes Metab. 2003;29:455-66

9. Codinach HP, Freixa PR. Diabetic cardiomyopathy: concept, heart function, and pathogenesis. An Med Interna 2002;19:313-20.

10. Brown HB, Waugh NR, J ennings PE. Microangiopathy as a prognostic indicator in diabetic patients suffering from acute myocardial infarction. ScottMedJ 1992; 37:44-6.

11. Bauters C, Lamblin N, Mc Fadden EP, Van Belle E, Millaire A, De Groote P. Influence of diabetes mellitus on heart failure risk and outcome. Cardiovascular Diabetology 2003,2:1.

12. Wolfenbuttel $B$, Boulanger $C$, Crijns FR et al. Breakers of advanced glycation end products restore large artery properties in experimental diabetes. Proc $\mathrm{Natl} A c a d$ SciUSA 1998; 95:4630-4.

13. Chaves FR, J orge PAR. Miocardiopatia diabética. Arq Bras Endocrinol Metab $1998 ; 42: 134-9$.

14. Gutierrez PS, Higuchi ML. Alterações cardíacas e vasculares no diabete: aspectos anatomopatológicos. Rev Soc Cardiol Estado São Paulo 1998;8:1020-4.

15. Rossen J D. Abnormal microvascular function in diabetes: relationship to diabetic cardiomyopathy. Coron Artery Dis 1996;7:133-8.
16. Devereux RB, Roman MJ, Paranicas M et al. Impact of diabetes on cardiac struture and function; the strong heart study. Circulation 2000;101:2271-6.

17. Zarich SW, Arbuckle BE, Cohen LR. Diastolic abnormalities in young asymptomatic diabetic patients assessed by pulsed Doppler echocardiography. J Am Coll Cardiol 1988;12:114-20.

18. Nitenberg A, Ledoux S, Valensi P, Sachs R, Attali J R, Antony I. Impairment of coronary microvascular dilatation in response to cold pressor-induced symphatetic stimulation in type 2 diabetic patients with abnormal stress thallium imaging. Diabetes 2001;50:1180-5.

19. Di Bonito P, Cuomo S, Moio N, Sibilio G, Sabatini D, Capa B. Diastolic dysfunction in patients with non-insulin-dependent diabetes mellitus of short duration. Diabet Med 1996; 13: 321-4.

20. Annonu AK, Fattah AA, Mokhtar MS, Ghareeb S, Elhendy A. Left ventricular systolic and diastolic functional abnormalities asymptomatic patients with non-insulin-dependent diabetes mellitus. J Am Soc Echocardiogr 2001;14:885-91.

21. LiuJ E, Robbins DC, Palmieri V et al. Association of albuminuria with systolic and diastolic left ventricular dysfunction in type 2 diabetes. The Strong Heart Study. J Am Coll Cardiol 2003;41:2022-8.

22. Bell DSH. Diabetic Cardiomyopathy. Diabetes Care 2003;26:2949-50.

23. American Diabetes Association. Clinical Practice Recommendations 1995. Screening for diabetic retinopathy. Diabetes Care 1995;18:21-3.

24. Sahn DJ, DeMaria A, Kisslo J, Weyman A. Recommendations regarding quantitation in M-mode echocardiography: results of a survey of echocardiographic measurements. Circulation 1978;58:1072-83.

25. Rakowski H, Appleton C, Chan Kl et al. Canadian consensus recommendations for the measurement and reporting of diastolic dysfunction by echocardiography.J Am Soc Echocardiogr 1996; 9: 736-60.

26. Mustonen J N, Uusitupa MI, Tahavanainen K et al. Impaired left ventricular systolic function during exercise in middle-aged insulin-dependent and noninsulin-dependent diabetic subjects without clinically evident cardiovascular disease. Am J Cardiol 1988;62:1273-9

27. Tischler MD. Clinical abnormalities of cardiac function and echocardiographic tissue characterization in diabetes mellitus. Coron Artery Dis 1996;7:139-42.

28. Struthers AD, Morris AD. Screening for and treating left-ventricular abnormalities in diabetes mellitus: a new way of reducing cardiac deaths. Lancet 2002;359: 1430-32.

29. Tenembaum A, Fisman EZ, Schwammenthal E, Adler Y, Benderly M, Shemesh J . 
Increased prevalence of left ventricular hypertrophy in hipertensive women with type 2 diabetes mellitus.Cardiovasc Diabetol 2003;2:14.

30. Galvan AQ, Galetta F, Natali A et al. Insulin resistance and hyperinsulinemia: No independent relation to left ventricular mass in humans. Circulation 2000;102:2233-8.

31. Rutter MK, Parise H, Benjamin EJ et al. Impact of glucose intolerance and insulin resistance on cardiac structure and function: sex-related differences in the Framingham Heart Study. Circulation 2003;107:448-54.

32. Zabalgoitia M, Ismaeil MF, Anderson L, Maklady FA. Prevalence of diastolic dysfunction in normotensive, asymptomatic patients with well-controlled type 2 diabetes mellitus. Am J Cardiol 2001;87: 320-3.

33. Schannwell CM, Schneppenheim M, Perings S, Plehn G, Strauer BE. Left ventricular diastolic dysfunction as an early manifestation of diabetic cardiomyopathy. Cardiology 2002; 98: 33-9.
34. Kannel W, Hjortland M, Castelli WP. Role of diabetes in congestive heart failure: the Framingham study. Am J Cardiol 1974;34:29-34

35. Fang ZY, Najos-Valencia O, Leano R, Marwick TH. Patients with early diabetic heart disease demonstrate a normal myocardial response to dobutamine. J Am Coll Cardiol 2003; 42: 451-2.

36. Poirier P, Garneau C, Bogaty P et al. Impact of left ventricular diastolic dysfunction on maximal treadmill performance in normotensive subjects with well controlled type 2 diabetes mellitus. Am J Cardiol 2000;85:473-7.

37. Picano E. Diabetic Cardiomyopathy: the importance of being earliest. J Am Coll Cardiol 2003;42:454-6.

38. Liu J, Masurekar MR, Vatner DE et al. Glycation end-product cross-link breaker reduces collagen and improves cardiac function in aging diabetic heart. Am J Physiol Heart Circ Physiol 2003;285:2587-91. 